\title{
Desafíos de la inclusión en la Educación Superior: reflexiones internacionales sobre prácticas educativas transformadoras
}

\author{
Desafios da inclusão no Ensino Superior: reflexões internacionais sobre \\ práticas educativas transformadoras
}

Challenges of inclusion in Higher Education: international reflections on transforming educational practices

\section{Eladio Sebastián-Heredero}

Doctor por la Universidad de Alcalá, Alcalá de Henares, Espanha.

eladio.sebastian@gmail.com

ORCID - http://orcid.org/0000-0003-0293-4395

\section{Patricia Tanganelli Lara}

Pós-doctoranda en la Universidade Federal de Mato Grosso do Sul, Campo Grande, Mato Grosso do Sul, Brasil. patriciatanganelli@gmail.com

ORCID - http://orcid.org/0000-0002-4616-4452

Recebido em 17 de julho 2020

Aprovado em 30 de julho de 2020

Publicado em 17 de novembro de 2020

\section{RESUMEN}

El objetivo de este artículo es presentar las políticas públicas puestas en marcha, tanto en España como en Brasil, para el apoyo al acceso y permanencia de estudiantes con discapacidad, incluyendo aquellos con trastorno del espectro autista, superdotados y/o altas habilidades, en instituciones públicas de Educación Superior. Para eso, utilizamos el método comparativo de dos realidades para ver sus semejanzas y diferencias e interpretar como en cada uno de los contextos se producen procesos de cambios desde la vertiente más interpretativa de las ciencias sociales. Los resultados apuntan que en ambos casos existe un reconocimiento y atención a los estudiantes con discapacidad en la enseñanza, implementado con legislación concreta al hilo de las recomendaciones de la UNESCO. Se observa que tanto en Brasil como en España existe una normativa específica sobre el acceso y permanencia de estudiantes en la Universidad; siendo que en España es exclusivo para las personas con discapacidad en tanto que en Brasil es compartido con otras diversidades y, por último, se constata que la regulación de los servicios para su implementación es más detallada con legislación en Brasil en tanto que en España queda en manos de la autonomía de las Universidades a partir de un marco regulador amplio.

Palabras clave: Inclusión en Educación Superior; políticas afirmativas; acceso a Educación Superior.

\section{RESUMO}

O objetivo desse artigo é apresentar as políticas públicas implementadas na Espanha e no Brasil, para o apoio ao acesso e a permanência de estudantes com deficiência, incluindo aqueles com transtorno do espectro autista e/ou altas habilidades, em instituições públicas de Ensino Superior. Para tanto, utilizamos como metodologia a pesquisa comparada, por 
http://dx.doi.org/10.5902/1984686X48224

analisar duas realidades que apresentam semelhanças e diferenças em decorrência do tempo e do espaço geográfico. Os resultados apontam que as normativas que regulam o processo de inclusão em ambos os países são muito parecidas, pois tem a origem nas orientações mundiais dadas pelas declarações da UNESCO. Observa-se que em ambos os casos existe uma legislação específica sobre o acesso e permanência dos estudantes na universidade, sendo que na Espanha é exclusiva para pessoas com deficiência enquanto que no Brasil é compartilhado com outras diversidades e, finalmente, fica constatado que a regulação dos serviços é mais específica na legislação brasileira e que na Espanha fica sob a responsabilidade da autonomia das universidades a partir de um marco regulador amplo.

Palavras-chave: Inclusão na Educação Superior; políticas afirmativas, Acesso à Educação Superior.

\section{ABSTRACT}

The objective of this article is to present the public policies put in place both in Spain and in Brazil, to support the access and stay of students with disabilities, including those with autism spectrum disorder, gifted and / or highly skilled, in institutions Public Higher Education. For this, we use the comparative method of two realities to see their similarities and differences, and to interpret how in each of the contexts processes of change take place from the most interpretive side of the social sciences. The results indicate that in both cases there is recognition and attention to students with teaching deficiencies in both countries implemented in line with the UNESCO recommendations. It is observed that in both cases there is a specific regulation on the access and permanence of students at the University that in Spain is exclusive for people with disabilities, while in Brazil it is shared with other diversities and, finally, it is verified that the regulation of services is more detailed with legislation in Brazil, while in Spain it remains in the hands of the autonomy of the Universities based on a broad regulatory framework.

Keywords: Inclusion in Higher Education; affirmative policies; access to Higher Education.

\section{Introducción}

La escolarización de estudiantes con discapacidad ${ }^{1}$, incluyendo aquellos con trastorno del espectro autista, superdotados y/o altas habilidades, en clases regulares, no puede ser considerada como un movimiento nuevo en Educación, el derecho al acceso y permanencia en estas aulas, en todos los niveles, fue regulado tras el movimiento político de educación para todos, en un ambiente inclusivo, que consideraba la diversidad de la población y sus necesidades.

Escolarmente hablando, la educación inclusiva asume un papel transformador y generador de nuevas prácticas, reflexivas, permitiendo que toda la comunidad educativa participe de los debates sobre la necesidad de abordarlas para lograr una educación de calidad que considere la diversidad. 
Hasta la década de los 90 del siglo XX, la escolarización de las personas con discapacidad estaba orientada con una perspectiva psico-médica. Según Laplane (2010, p.19), la perspectiva psico-médica "se refiere a un sistema de ideas centrado en el déficit y en las carencias. Una de las principales marcas de esa concepción fue entender la discapacidad como algo inherente al propio individuo". En ese momento la segregación de las personas con discapacidad era la marca de la Educación Especial, confiadas a pocos profesionales e ligadas a instituciones médico patológicas, que se preocupaban de la rehabilitación de la anormalidad o la minimización de los daños causados por ella.

Mundialmente desde ese momento hubo muchas conquistas sobre estos derechos a partir de eventos como la Conferencia Mundial sobre Educación para Todos, Jomtien 1990, y la Declaración de Salamanca en 1994, realizada en el seno de la Conferencia Mundial de Educación Especial y ambas firmadas por los gobiernos de España y Brasil y que llevó a grandes cambios en sus sistemas educativos para la inclusión de las personas con discapacidad.

El tema de la inclusión continuó teniendo un destaque importante desde el inicio del siglo XXI iniciado con el Fórum Educativo Mundial, Dakar 2000. En 2006 Brasil y España participaron en otro de marco general, fue la Convención Internacional sobre los Derechos de las Personas con Deficiencia, Nova York, donde se elaboró el documento que defiende el derecho de garantizar la educación para todas estas personas, firmado por España ese mismo año y por Brasil en 2009. A estas siguieron la Declaración de Lisboa (2007) y la Conferencia Internacional de Educación de Ginebra (2008). En todos estos encuentros hubo un gran avance en cuanto a los apoyos necesarios para la inclusión con el objetivo de ecualizar los derechos para todos y estos referentes son asumidos internacionalmente, por lo que partimos de un punto común.

Con foco en esta investigación en 1998 se desarrolló la Declaración Mundial sobre Educación Superior en Paris, que fue una inspiración para las legislaciones de Brasil, España y del mundo, buscando garantizar a las personas con discapacidad el derecho al acceso a la Educación Superior, también inclusiva (UNESCO, 1998).

En 2009 se realizó la Segunda Conferencia Mundial de Educación Superior, Paris, (UNESCO, 2009) y aunque no se tocó específicamente el tema de la discapacidad se explicita la necesidad de buscar equidad y calidad en la Educación Superior, y de forma implícita está recogido que sea para todos y todas independientemente de sus condiciones personales. 
http://dx.doi.org/10.5902/1984686X48224

Las condiciones de acceso y permanencia de los estudiantes con discapacidad en la Educación Superior han sido muy investigadas (VELOSO e MACIEL, 2015; COUZENS et al, 2015; BUTLER et al, 2016; SILVA e CAMARGO, 2018; CASTRO e AMARAL, 2018) presentando desde las actitudes sociales a los apoyos necesarios en el proceso de inclusión de las personas con discapacidad en la Educación Superior algo que ha evolucionado significativamente en los últimos años.

De acuerdo con el Censo de la Educación Superior de 2018 (INEP, 2019) en Brasil en 2018 había un total de 2537 instituciones de educación superior, agrupadas en Universidades, 107 públicas y 92 privadas; Centros Universitarios de ellos 13 públicos y 217 privados; Facultades públicas 139 y privadas 1929 y 40 Institutos Federales. El total de alumnos matriculados en todas ellas ese año era de 8.450.755 con un aumento progresivo y constante la población universitaria en Brasil a lo largo de estos últimos años, desde los poco más de 7 millones en 2012.

En lo que se refiere al número de matrículas en Cursos de Graduación de alumnos con discapacidad, trastornos globales del desarrollo o altas habilidades/superdotación, ha habido un incremento pasando de los $0,37 \%$ de la población estudiantil de la Educación Superior que suponían los 26.663 alumnos en 2012 al 0,51\% en el año de 2018 con 43.633 alumnos con discapacidad de la población universitaria brasileña (INEP, 2019).

Conforme datos del Ministerio de Educación Español (ESPAÑA, 2019), el Sistema Universitario Español (SUE), en el curso 2017-2018, tenía un total de 84 Universidades, no existe otro tipo de institución de Educación Superior, siendo 82 en actividad, con 50 públicas y 32 privadas. Ese curso estaban matriculados en las Universidades Españolas 1.484.741 estudiantes, en los cursos de Graduación, $1^{\circ}$ e 2ํㅡㄹ Ciclo, Máster y Doctorados.

En España (UNIVERSIA, 2019) también ha habido un importante aumento de matrículas universitarias en este periodo estudiado, del 1,1 millón de estudiantes en 2012 a casi 1,5 millones de universitarios en 2018. La evolución de los alumnos con discapacidad en España también se multiplicó, en 2012 había 12.755 lo que suponía el 1,1\% del total, en tanto que en 2018 llegó a 21.435 el 1,5\% según el IV Informe sobre la Educación Superior en el que participaron 65 universidades.

Como podemos ver al analizar estos datos, ambos países, considerando las diferencias de contexto y del proceso de inclusión de los estudiantes en la Educación Superior, han tenido un comportamiento similar con aumento de las matrículas en la última década en torno al $20 \%$ y un incremento de la entrada de alumnos con discapacidad en 
http://dx.doi.org/10.5902/1984686X48224

ellas de más del 70\%, si bien el porcentaje de alumnos declarados con discapacidad incluidos en las universidades en ambos países es bastante diferente fruto de las diferentes trayectorias de ambos contextos, no obstante estamos con Omote (2016, p. 212) cuando resalta que

La enseñanza de calidad, con todos los recursos necesarios para el total aprovechamiento por parte de todos los estudiantes, incluyendo aquellos con deficiencia, es uno de los ingredientes para definir la Enseñanza universitaria como inclusiva. Los estudiantes con deficiencia, así formados, precisan estar aptos para acceder al mercado de trabajo en condiciones de igualdad con cualquier persona con la misma formación. La necesidad de cotas para el ingreso de personas con deficiencia en el mercado puede ser interpretada, en parte, como fracaso de la educación inclusiva.

Estos dos aspectos incremento progresivo de matrículas e importante avance en el acceso de estudiantes con discapacidad en la Educación Superior, sin duda, justifica este estudio.

Además del acceso tenemos que considerar también las barreras físicas, pedagógicas, arquitectónicas, comunicativas, emocionales y actitudinales que existen y que, de alguna manera, creemos que pretenden ser abordadas y superadas desde las políticas. Ese tema es discutido por López-Gavira y Moriña-Díez (2015) o André y Ribeiro (2018) como un proceso de invisibilidad, percibido por estudiantes con discapacidad. Así nos hacemos eco de las palabras de Moriña-Díez y Carballo (2020) cuando afirman en sus conclusiones que reconocer la existencia de necesidades, así como tener en cuenta propuestas, a partir de otras investigaciones, podría contribuir al desafío de desarrollar políticas y prácticas que contribuyan a construir una universidad más inclusiva y para todos.

Para concluir traemos las palabras de Sebastián-Heredero (2010) cuando alertan de que al hablar de Educación Inclusiva es necesario reflexionar sobre el proceso de reconstrucción escolar relativo a la innovación y mejora de las prácticas, que vienen determinadas inicialmente a partir de políticas, con el objetivo de promover la real participación y, sobre todo, el rendimiento de todos los estudiantes.

Con el objetivo de analizar las prácticas para la inclusión de estudiantes con discapacidad en la Educación Superior, desarrolladas en Brasil y en España, este trabajo presenta inicialmente un estudio comparativo de las políticas públicas promovidas en estos países, para el apoyo al acceso y permanencia de los mismos en instituciones de Educación Superior, así como para el desarrollo de acciones con estos mismos fines. 
http://dx.doi.org/10.5902/1984686X48224

\section{Metodologia}

El método comparativo es el utilizado en esta investigación en el sentido dado por Skocpol e Somers (1980, apud Collier, 1994), cuando afirman que puede ser utilizado para un número pequeño de casos y aunque exista el problema de tener más variables que casos, en cualquier caso permite hacer análisis sistemáticos que, cuando utilizados correctamente, nos sirven para confrontar datos. En este caso en concreto comparando dos casos, a través de sus desarrollos legislativos, para ver sus diferencias recíprocas e interpretar como en cada uno de los contextos se producen procesos de cambios desde la vertiente más interpretativa de las ciencias sociales.

Para este estudio comparativo hacemos de él un procedimiento sistemático por el que contrastamos el fenómeno legislativo y a través de él establecemos similitudes y diferencias entre ellos. A partir de estos presupuestos profundizamos en el conocimiento de las políticas públicas para el acceso y permanencia de estudiantes de Educación Especial/discapacidad en la Educación Superior, desde el análisis documental de fuentes primarias de las publicaciones legislativas hechas en cada país. Franco (2000) considera que hacer analogías y comparar son procesos inherentes al acto de conocer y de construir un discurso sobre la realidad que será nuestra forma de análisis de los datos.

El criterio de selección de los instrumentos legislativos de cada país parte de la promulgación de sus Constituciones, transitando por las leyes generales de Educación en ambos contextos y todas las que se refieren a la inclusión de estudiantes con discapacidad en la Educación Superior, llegando a los decretos, resoluciones, portarías, circulares y recomendaciones que instituyen el desarrollo de acciones concretas para el acceso y permanencia, así como la instauración de servicios de apoyo a los estudiantes.

Para una mejor comprensión y visualización de los documentos analizados en ambos países presentamos una línea cronológica paralela referente a las dos realidades estudiadas. 
http://dx.doi.org/10.5902/1984686X48224

Figura 1 - Línea cronológica con la disposición de los documentos de Brasil y España sobre el acceso de los estudiantes con discapacidad en la Educación Superior.

\begin{tabular}{|c|c|c|c|c|c|}
\hline BRASIL & & 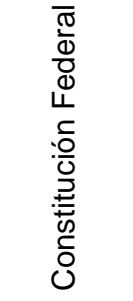 & 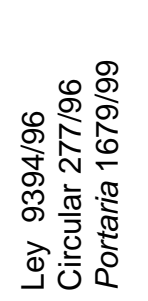 & 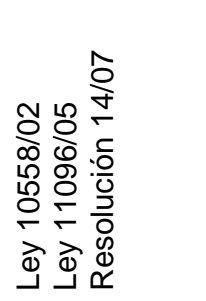 & $\begin{array}{l}\frac{10}{0} \\
0 \\
0 \\
\sigma\end{array}$ \\
\hline Década & $1970-79$ & 1980-89 & $1990-99$ & $2000-2009$ & $2010-2020$ \\
\hline
\end{tabular}

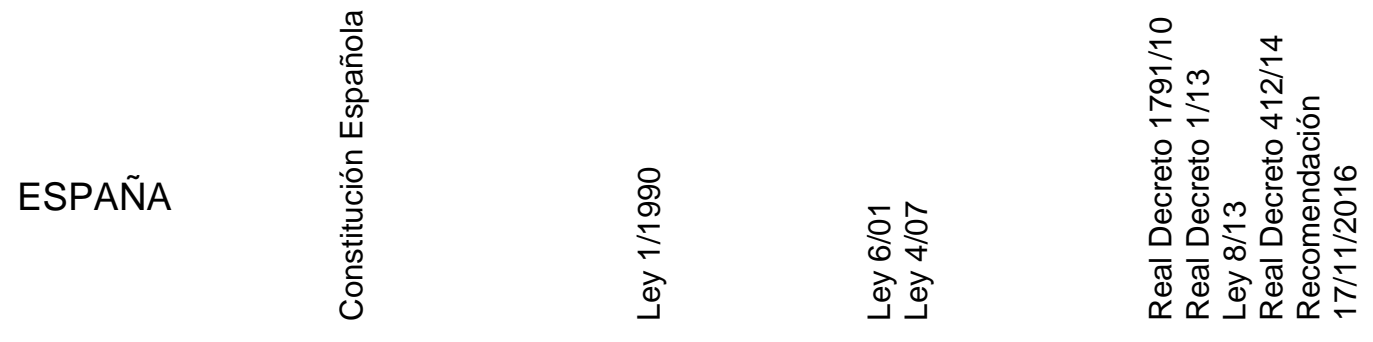

Fuente: elaboración propia (2020).

Estas nos sirven para trabajar las variables de análisis del estudio que serían: reconocimiento y atención a los estudiantes con discapacidad en la Educación Superior en las normas de carácter general; legislación específica para el acceso y permanencia en estas Instituciones y regulación de los servicios de apoyo al estudiante con discapacidad en ella.

Las legislaciones se vuelven transcendentes en tanto que las semejanzas y diferencias de las políticas públicas implementadas en estos países, además de los hechos analizados demuestran la trayectoria cultural e histórica de los espacios que ocupan. Franco (2000, p.197) afirma que "procurar conocer las diferentes soluciones que otros países y otros pueblos dan a sus problemas, a sus instituciones, como en lo que se refiere a la educación, siempre fue un medio de desarrollo y de enriquecimiento".

\section{Políticas Públicas de acceso a la Educación Superior en Brasil para personas con discapacidad}

En Brasil, la Constitución Federal de 1988 (BRASIL, 1988), artículo 205, considera la educación como deber del Estado y de la familia, sin distinción de público y de su condición 
http://dx.doi.org/10.5902/1984686X48224

física o intelectual y el artículo 208, punto IV, reconoce el acceso a los niveles más elevados de enseñanza, de investigación y de creación artística, según la capacidad de cada uno.

La Ley de Directrices y Bases de la Educación, LDB, (BRASIL, 1996a), regula que la Enseñanza debe ser organizada con base en el principio de la igualdad de condiciones para o acceso y permanencia en las instituciones educativas, garantizando a las personas con discapacidad el derecho a la matricula preferencialmente, en las instituciones ordinarias y la atención educativa especializada y gratuita. Además, dice que los sistemas educativos garantizarán a estudiantes con discapacidad currículos, métodos, técnicas, recursos educativos y organización específicos, para atender sus necesidades.

Las implicaciones para la educación inclusiva, demandarán la transformación del ambiente educativo y la movilización de todos los relacionados en el proceso. El mismo año de publicación de la LDB se emitió la Circular 277/MEC/GM (BRASIL, 1996b), que hace referencia a la atención de las necesidades de los alumnos con discapacidad visual, física y auditiva, recomendando los ajustes en el proceso de selección de esos estudiantes en la Educación Superior (IES). Esa recomendación no determinaba unas pautas para la permanencia en ese nivel de enseñanza, se centraba en la ejecución de una política adecuada en el sentido de "adecuarse, estructuralmente, para crear condiciones propias, de forma que se haga posible su acceso a la Educación Superior" (BRASIL, 1996b).

En 1999 es publicada la Portaria 1679 (BRASIL, 1999), con el objetivo de instruir los procesos de autorización y de reconocimiento de cursos, y de acreditación de instituciones, estableciendo los requisitos de accesibilidad a la Enseñanza de personas con discapacidad física y sensorial. En 2003 ésta fue derogada y publicada otra, con nuevo texto, al objeto hacerlo viable en función de las necesidades para la implementación de lo propuesto.

Las políticas desarrolladas por el Gobierno Federal brasileño, en lo que se refiere a la Educación Superior, en los años siguientes, fueron dirigidas a las necesidades y a atención prioritaria. En 2002, el Programa Diversidad en la Universidad, creado por la Ley 10.558 (BRASIL, 2002), estableció la Ley de Reserva da Plazas (Cotas), con la finalidad de mejorar y evaluar estrategias para la promoción del acceso a la Educación Superior de personas pertenecientes a grupos socialmente desfavorecidos. A pesar del Programa citar especialmente a los afro descendientes y a los indígenas brasileños, las personas con discapacidad también estaban contempladas en el grupo de personas socialmente desfavorecidas. 
http://dx.doi.org/10.5902/1984686X48224

Sobre este asunto, Branco, Jezine y Nakamura (2016, apud CABRAL, 2018) resaltan que "las políticas de educación superior con foco en la ampliación del acceso señalan la perspectiva de inclusión de los sujetos históricamente excluidos de ese nivel de enseñanza, como negros, mujeres, deficientes, alumnos de escuela pública y de rentas bajas" (pp. 260261).

Las políticas públicas de acciones de igualdad (afirmativas) tienen un origen difuso e históricamente construido, como afirma Cabral (2018), y avanzan de forma más intensa desde este momento de perspectiva inclusiva.

Cuando profundizamos sobre la garantía de igualdad de derechos, no siempre nos liberamos de una percepción heredada históricamente en la cual ese principio ha sido predominantemente comprendido, a lo largo de los últimos siglos, como la mera separación de privilegios, exenciones personales y regalías de clase (p. 10-11)

Así destacamos la publicación de la Ley no 11.096 (BRASIL, 2005), que amplió el acceso y la permanencia de las personas con discapacidad a la Educación Superior. Esta ley creó el Programa Universidad para Todos - PROUNI, reservando un porcentaje de becas para las personas con discapacidad en universidades privadas, desde que respondan a los criterios establecidos en el programa.

En 2005 se creó el Programa Incluir: Accesibilidad en la Educación Superior para las Instituciones Federales de Enseñanza Superior, y en 2007 se publicó la Resolución 14 (BRASIL, 2007a), donde en su artículo primero destaca la necesidad de que las Instituciones de Enseñanza Superior promuevan acciones para garantizar el acceso total a las personas con discapacidad, haciendo accesible el "ambiente físico, portales y webs electrónicos, los procesos selectivos, las prácticas educativas, las comunicaciones y las evaluaciones, dando respuestas concretas a las diferentes formas de exclusión" (p. 1).

Anache, Rovetto y Oliveira (2014), sobre esta cuestión, resaltan la importancia que tiene mover las estructuras institucionales y cuestionar la cultura de la homogenización con revisión de procesos de matrícula, tipo de elección profesional, organización del currículo, formas de evaluación, "llevándonos a debatir normas para la construcción de proyectos de enseñanza para las Instituciones de Enseñanza Superior, en la perspectiva de la educación inclusiva" (p. 309).

En 2007, el Plan de Desarrollo de Educación - PDE, tuvo como objetivo mejorar la educación en el país, en todas sus etapas, en un plazo de quince años, y presentó en uno 
http://dx.doi.org/10.5902/1984686X48224

de sus ejes, el acceso y permanencia de las personas con discapacidad en la Educación Superior, en uno de sus principios fundamentales se destaca:

i) expansión de la oferta de plazas, [...], ii) garantía de calidad, [...], iii) promoción de inclusión social por la educación, [...], iv) ordenación territorial, permitiendo que la Enseñanza de calidad sea accesible a las regiones más remotas del País, e v) desarrollo económico y social, haciendo que la educación superior, sea un su parte de formadora de recursos humanos altamente cualificados, sea como pieza imprescindible en la producción científico-tecnológica, elemento clave de integración y de formación de la Nación (BRASIL, 2007b, p.15).

La perspectiva de Educación Inclusiva en Brasil, para los estudiantes con discapacidad, fue gradualmente desarrollada en la Educación Superior y tuvo su marco de garantías, de derechos de acceso y permanencia, por medio de la publicación de la Política Nacional de Educación Especial desde la Perspectiva de Educación Inclusiva (BRASIL, 2008). Ésta cita la "transversalidad de la modalidad de educación especial desde la educación infantil hasta la Educación Superior" (BRASIL, 2008, p.14).

En 2015, se promulgó la Ley 13.146 de Inclusión de la Persona con Deficiencia (BRASIL, 2015), reafirmando el derecho a la educación, tratando de los procesos selectivos, de ingreso y permanencia en IES y de educación profesional y tecnológica, privadas y públicas, exclusivamente para las Instituciones Federales de Enseñanza, la Ley 13.409, (BRASIL, 2016), prevé la reserva de plazas para personas con discapacidad en cursos técnicos de nivel medio y superior.

\section{Políticas Públicas de acceso a la Educación Superior en España para personas con discapacidad}

La Constitución Española de 1978 (ESPAÑA, 1978) en su artículo 49, estableció que los poderes públicos deben realizar una política de previsión, tratamiento, rehabilitación e integración en favor de las personas con discapacidad física, sensorial y psíquica, debiendo prestar la atención especializada que precisan lo que abarca a la inclusión social y educativa.

El derecho a la educación y la atención educativa a las personas con discapacidad expresado en la Constitución de 1978 se desarrolló con la Ley Orgánica General del Sistema Educativo, LOGSE, (ESPAÑA, 1990), destacando en su Capítulo V que el sistema educativo dispondrá de los recursos necesarios para atender a los 
estudiantes con necesidades educativas especiales. La Ley, siguiendo el movimiento histórico, tenía como principio la normalización y la integración escolar.

Posteriormente fue promulgado el reglamento educativo vigente actualmente que es la Ley Orgánica para la Mejora de la Calidad Educativa (ESPAÑA, 2013a), que tiene entre sus objetivos promover o máximo desarrollo personal y profesional de las personas y para su desarrollo normativo serán las Comunidades Autónomas las encargadas de hacerlo y sin avance significativo en lo que se refiere a inclusión escolar.

En lo que se refiere a la Educación Superior la Ley Orgánica de Universidades (ESPAÑA, 2001) fue un referente en lo que se refiere al acceso de personas con discapacidad y para ello tiene una Disposición adicional, vigésima cuarta, que trata específicamente de la inclusión de las personas con discapacidad en las universidades, a partir de lo expresado en el artículo 46.2.b:

La igualdad de oportunidades y no discriminación por razones de sexo, raza, religión o discapacidad o cualquier otra condición o circunstancia personal o social en el acceso a la universidad, ingreso en los centros, permanencia en la universidad y ejercicio de sus derechos académicos. (ESPAÑA, 2001, $p$ 30)

La nueva Ley 4/2007 Orgánica de Universidades, (ESPAÑA, 2007) fue la propuesta para dar secuencia y en ella se establece que las personas con discapacidad deben recibir ayuda personalizada, apoyos y adaptaciones de los profesores para su participación plena y efectiva, no pudiendo ser discriminadas en se acceso, ingreso, permanencia y ejercicio profesional con los títulos académicos.

Para complementar esta mirada específica se publicó el Real Decreto 1791 (ESPAÑA, 2010) por el que se aprueba el Estatuto del Estudiante Universitario en el que entre otras cosas se habla del acceso y admisión y de su acompañamiento dentro de la institución además de la no discriminación, de la necesidad de hacer adaptaciones y establecer recursos e indicando el derecho a la no discriminación por razón de discapacidad o por motivos de enfermedad. El Decreto establece que las universidades deberán crear acciones necesarias para garantizar que los estudiantes puedan conseguir los conocimientos y competencias académicas y profesionales. Entre ellas se destacan la creación y desarrollo de servicios para los estudiantes universitarios con discapacidad, que permitan proporcionar los recursos y apoyos necesarios para garantizar la igualdad de oportunidades. 
http://dx.doi.org/10.5902/1984686X48224

En este sentido Alarcón (2014) dice que los principales ajustes para la inclusión son: adaptaciones de las pruebas de acceso a la universidad; reserva de $3 \%$ de las matrículas para estudiantes que presenten un grado de discapacidad, legalmente reconocida, recursos, apoyos y servicios con el objetivo de favorecer el acceso y permanencia de sus estudios. No obstante fue necesario establecer la Recomendación 17/11/2016 de defensor del pueblo por la que se instaba a las Universidades a reserva un número de plazas para las personas con discapacidad, también en la postgraduación.

Es fundamental destacar el Real Decreto 1 (ESPAÑA, 2013b) por el que se aprueba el Texto Refundido de la Ley General de derechos de las personas con discapacidad y de su inclusión social y en su artículo 20.C se dice expresamente:

\begin{abstract}
Las personas que cursen estudios universitarios, cuya discapacidad les dificulte gravemente la adaptación al régimen de convocatorias establecido con carácter general, podrán solicitar y las universidades habrán de conceder, de acuerdo con lo que dispongan sus correspondientes normas de permanencia que, en todo caso, deberán tener en cuenta la situación de las personas con discapacidad que cursen estudios en la universidad, la ampliación del número de las mismas en la medida que compense su dificultad, sin mengua del nivel exigido. Las pruebas se adaptarán, en su caso, a las características de la discapacidad que presente el interesado (ESPAÑA, 2013b, p. 36)
\end{abstract}

Por último, el Real Decreto 412 (ESPAÑA, 2014) de 6 de junio, establece la normativa básica de los procedimientos de ingreso a los Centros Universitarios Oficiales de Graduación, reconociendo y ordenando que las pruebas deben ser adaptadas, según las necesidades de cada aluno.

Al igual que decíamos anteriormente la legislación específica sobre la organización y ordenamiento de las Universidades está bajo la competencia de las Comunidades Autónomas y por tanto existen muchas características específicas según la región, no obstante es común que todas las Universidades Públicas y la mayoría de las privadas tengan un Servicio de Apoyo a las personas con discapacidad.

El acceso a la Educación Superior considera las necesidades físicas, intelectuales, sociales, emocionales, lingüísticas y otras, que fundamentan una educación para todos, estableciendo que dentro de la autonomía de las Universidades deben establecer estrategias de acceso al conocimiento.

Según Alegre-Sánchez, et al (2019) la actual legislación recurre a medidas positivas como instalación, en las Universidades, de un sistema de tutoría y orientación personalizada a los estudiantes con discapacidad, para la planificación de estrategias que 
http://dx.doi.org/10.5902/1984686X48224

favorezcan la trayectoria educativa y su permanencia durante toda la graduación. Estas acciones contribuyen con el principio de igualdad de oportunidades y reflejan el compromiso con la calidad educativa.

Por su parte Alegre-Sánchez, et al (2019) manifiestan que la aprobación y los avances legislativos cuentan con la participación activa de las organizaciones representativas de los sectores de las personas con discapacidad, que han desarrollado un papel fundamental para los avances y garantía de los derechos de esas personas.

Derivado también de la legislación general sobre Universidades (ESPAÑA, 2001, 2007) exige de las universidades la implantación de medidas contra la discriminación y acciones positivas, y además de establecer programas de apoyo, proporcionar recursos y realizar adaptaciones y procedimientos con objetivos y garantías de los principios de igualdad de oportunidades y condiciones, de no discriminación, también trabajar dentro de los parámetros de un diseño universal de accesibilidad para todos.

Las Universidades españolas gozan de autonomía para responder a las necesidades, y pueden presentar formas diferentes de actuación con sus servicios y programas dirigidos a los estudiantes con discapacidad que además tienen sistemas de control y evaluación propios en muchos casos.

\section{Consideraciones finales}

En el ámbito de las Políticas de acceso y permanencia de estudiantes con discapacidad en instituciones de Educación Superior en Brasil y España, observamos algunas semejanzas y diferencias, justificadas por sus realidades y contextos diferentes a partir de la comparación de su normativa.

Los avances con la publicación de leyes, que reconocen y garantizan el derecho al acceso sin discriminación están presentes en los dos países, fundamentalmente después de la publicación de sus Constituciones democráticas y con legislaciones que fundamentaron e desarrollaron diversas acciones de igualdad (afirmativas).

Las normativas que regulan el proceso de inclusión en ambos países son muy parecidas, pues tienen su origen en las orientaciones mundiales, declaraciones de la UNESCO, y siguen con instrumentos propios de cada país regulados por marcos generales, sus leyes de educación LDBN en Brasil y LOGSE y LOMCE en España, y marcos específicos para el acceso de las personas con discapacidad y para la inclusión en la 
http://dx.doi.org/10.5902/1984686X48224

Educación Superior Ley 10558/2002 de diversidad en la Universidad y Ley 4/2007 de Universidades en España.

La concepción de las dificultades de acceso y permanencia, a veces aparece como un problema en función de la discapacidad, sin embargo ha tenido una nueva visión a partir de la explicitación de derechos y relaciones con el ambiente y la formulación de estrategias, para ofrecer servicios y recursos de apoyo algo que aparece más claramente en la legislación española, la Ley 11096/2005 y la Ley 13409/2016 hablan específicamente del acceso a la Universidad en Brasil con la determinación de cotas, algo similar a lo que ocurre en España con el Real Decreto 412/2014 y con la Recomendación 17/11/2016 aunque en España estas se refieren exclusivamente a las personas con discapacidad.

La percepción de un distanciamiento entre las dos realidades, se observa en la exigencia, por el gobierno español de un plano de acción individual para estudiantes con discapacidad, en la Educación Superior, Real Decreto 1791/2010 para garantizar el principio de igualdad de oportunidades, algo que en Brasil ha ocurrido sólo en Educación Básica.

Así mismo en las acciones desarrolladas por las legislaciones española y brasileña se recoge la necesidad de crear servicios de apoyo a los estudiantes con discapacidad y en ambos aparecen; sin embargo, en tanto que en España, Ley 4/2007, estos servicios son creados, sostenidos y evaluados por las propias universidades en Brasil, Resolución 14/2007, dependen de recursos externos y muy focalizados que no siempre llegan a materializarse.

Por último, parece evidenciarse, en los análisis realizados, que aunque en ambos países las acciones en concreto son muy parecidas en España su reglamentación se da a partir de una ley marco y un desarrollo que entra dentro del campo de la propia universidad, en tanto que en Brasil existen una serie de disposiciones derivadas de la ley marco, Ley $4 / 2007$ y Ley 8/2013, que organizan todas esas acciones.

Los cambios y trasformaciones sociales demandan que se desarrolle la equidad con acciones de igualdad entre los alumnos y profesores, la creación de espacios más accesibles, la existencia de una corresponsabilidad individual y grupal para garantizar el aprendizaje de todos y por fin, un trabajo más colaborativo que considere la necesidad singular de cada uno, algo en desarrollo en ambos países.

Aunque no es objeto de este análisis específico somos conscientes de que las universidades de los dos países tienen, en función de las normas reguladoras de cada 
http://dx.doi.org/10.5902/1984686X48224

contexto, sus unidades de inclusión para organizar estructuralmente el día a día de las personas auto declaradas deficientes; pero quedan todavía muchos con dificultades de aprendizaje y/o discapacidad fuera de estas estructuras, que los podrían atender y mejorar la calidad de la enseñanza y de la vida personal de cada uno de ellos.

\section{Referências}

ALARCÓN, María Esther Barradas. Seguimiento de Egresados. EEUU: Palibrio, 2014.

ALEGRE-SÁNCHEZ, María Ángeles; AGUDO-ARROYO, Yolanda; VALLÈS-SEGALÉS, Antoni. Discapacidad, becas y logro educativo en la educación superior. Educación Inclusiva, 12(1), p. 183-204, 2019.

ANACHE, Alexandra Ayach; ROVETTO, Stella Maris Rovetto; OLIVEIRA, Regiane Alves de. Desafios da implantação do atendimento educacional especializado no Ensino Superior. Revista Educação Especial, Santa Maria, 27(49), p. 299-312, 2014. Disponível em: https://periodicos.ufsm.br/educacaoespecial/article/view/9037. Acesso em: 25 jun. 2020.

ANDRE, Bianka Pires; RIBEIRO, Ana Luiza Barcelos. A invisibilidade de alunos com deficiência no Ensino Superior do Norte Fluminense. In: MOL, Gerson de Souza; MELO, Douglas Christian Ferrari de. (Org.). Pessoas com deficiência no ensino superior: Desafios e possibilidades. Brasil: Multicultural, 2018. p. 70-81.

BRASIL. Constituição da República Federativa do Brasil. Brasília, Diário Oficial da União, n 191-A, p. 19597-19648, 5/10/1988.

BRASIL. Lei 9.394 de Diretrizes e Bases da Educação. Brasília: Diário Oficial da União, Seç 1, p. 28110-28143, 23/12/1996a.

BRASIL. Aviso Circular 277/ MEC/ GM, de 8 de maio de 1996. Dirigido aos Reitores das IES, solicitando a execução adequada de uma política dirigida aos portadores de necessidades especiais, Brasília: MEC. 1996b. Disponível em:

http://portal.mec.gov.br/seesp/arquivos/pdf/aviso277.pdf Acesso em 4 de mar. 2020.

BRASIL. Portaria 1679, de 2 de dezembro de 1999. Dispõe sobre requisitos de acessibilidade de pessoas portadoras de deficiências, para instruir os processos de autorização e de reconhecimento de cursos e de credenciamento de instituições. Brasília, MEC. 1999. Disponível em: http://portal.mec.gov.br/sesu/arquivos/pdf/c1_1679.pdf. Acesso em 4 mar. 2020.

BRASIL. Lei 10.558, de 13 de novembro de 2002. Cria o Programa Diversidade na Universidade, e dá outras providências. Brasília: Diário Oficial da União, p. 6, col. 1, $14 / 11 / 2002$. 
http://dx.doi.org/10.5902/1984686X48224

BRASIL. Lei 11.096, de 13 de janeiro de 2005. Institui o Programa Universidade para Todos -PROUNI- regula a atuação de entidades beneficentes de assistência social no ensino superior. Brasília: Diário Oficial da União, p. 13, seç. 1, 14/01/2005.

BRASIL. Portaria normativa 14, de 24 de abril de 2007. Dispõe sobre a criação do "Programa Incluir: Acessibilidade na Educação Superior. Brasília: Diário Oficial da União, p. 4, seç. 1, 26/04/2007a.

BRASIL. O Plano de Desenvolvimento da Educação: razões, princípios e programas PDE. Brasília: Diário Oficial da União, n. 78, p. 27, seç. 1, 24/04/2007b.

\section{BRASIL. Política Nacional de Educação Especial na Perspectiva da Educação}

Inclusiva. Brasília: MEC/SEESP, 2008. Disponível em:

http://portal.mec.gov.br/arquivos/pdf/politicaeducespecial.pdf. Acesso em 28 mar. 2020.

BRASIL. Plano Nacional de Educação 2014-2024. Brasília: Diário Oficial da União , p. 1, Edição extra, seç. 1, 26/06/2014.

BRASIL. Lei 13.146, de 6 de julho de 2015. Institui a Lei Brasileira de Inclusão das Pessoas com Deficiência (Estatuto da Pessoa com Deficiência). Brasília: Diário Oficial da União, p. 2, seç, 1, 7/07/2015.

BRASIL. Lei no 13.409, de 28 de dezembro de 2016, dispõe sobre a reserva de vagas para pessoas com deficiência nos cursos técnico de nível médio e superior das instituições federais de ensino. Brasília: Diário Oficial da União, p. 3, col. 3, 29/12/2016.

BUTLER, Mathew, et al. Understand the graphic challenges faced by visually impaired students at Australian universities. Higher Education Research e Development, n. 36(1), p. 59-72, 2016.

CABRAL, Leonardo Santos Amâncio (2018). Políticas de ações afirmativas, pessoas com deficiência e o reconhecimento das identidades e diferenças no ensino superior brasileiro. Arquivos Analíticos de Políticas Educativas, 26(57), p. 1-33, 2018.

CASTRO, Bianca Gomes da Silva Muylaert Monteiro de; AMARAL, Shirlena Campos de Souza. Ações afirmativas no ensino superior: a utilização das cotas para pessoas com deficiência na UENF. In: MOL, Gerson de Souza; MELO, Douglas Christian Ferrari de. (Org.). Pessoas com deficiência no ensino superior: Desafios e possibilidades. 1ed. Brasil: Multicultural, 2018. p. 90-107.

COLLIER, David. El método comparativo: dos décadas de cambio. In: SARTORI, Giovanni.; MORLINO, Leonardo. La comparación en las ciencias sociales. Madrid: Alianza, 1994. p. 51-80.

COUZENS, David et al. Apoyo a estudiantes con discapacidades ocultas en universidades: un estudio de caso. International Journal of Disability, Development and Education, n. 62(1), p. 24-41, 2015.

ESPAÑA. Constitución Española. Madrid: Boletín Oficial del Estado, n. 311.1, s/p, 29/12/1978. 
http://dx.doi.org/10.5902/1984686X48224

ESPAÑA. Ley 1/1990 Orgánica General del Sistema Educativo. Madrid, Boletín Oficial del Estado, n. 238, p. 28927-28942, 4/10/1990.

ESPAÑA. Ley 6/2001 Orgánica de Universidades. Madrid, Boletín Oficial del Estado, n. 307, p. 49400-49425, 24/12/2001.

ESPAÑA. Ley Orgánica 4/2007 por la que se modifica la Ley Orgánica de Universidades 6/2001. Madrid, Boletín Oficial del Estado, n. 89, p. 16450-16451, 13/04/2007.

ESPAÑA. Real Decreto 1791/2010, de 30 de diciembre, por el que se aprueba el Estatuto del Estudiante Universitario. Madrid, Boletín Oficial del Estado, n. 318, p. 95635-95673, 31/12/2010.

ESPAÑA. Ley 8/2013 Orgánica de Calidad y Mejora de la Educación. Madrid, Boletín Oficial del Estado, n. 295, p. 97858-97921, 10/12/2013a.

ESPAÑA. Real Decreto 1/2013, de 29 de noviembre, por el que se aprueba el Texto Refundido de la Ley General de derechos de las personas con discapacidad y de su inclusión social. Madrid, Boletín Oficial del Estado, n. 289, p. 28927-28942, 3/12/2013b.

ESPAÑA. Real Decreto 412/2014, de 6 de junio, por el que se establece la normativa básica de los procedimientos de admisión a las enseñanzas universitarias oficiales de Grado Madrid, Boletín Oficial del Estado, n. 138, p. 43307-43323, 7/06/2014.

ESPAÑA. Recomendación 17/11/2016 de defensor del pueblo por la que se instaba a las Universidades a reserva un número de plazas para las personas con discapacidad, también en la post-graduación. Madrid, Defensor del Pueblo. 2016. Disponible en:

https://www.defensordelpueblo.es/resoluciones/modificacion-normativa-basica-para-incluir-laobligacion-de-las-universidades-de-reservar-un-porcentaje-de-plazas-para-la-admision-aestudios-de-master-y-doctorado-de-los-estudiantes-afectados-de-disc/ Acesso em: 23 abr. 2020.

\section{ESPAÑA. Catálogo Oficial de indicadores del Sistema Integrado de Información} Universitaria 2019. Madrid, MECD. 2019.

FRANCO, Maria Ciavatta. Quando nós somos o outro: questões teórico-metodológicas sobre os estudos comparados. Educação e Sociedade. n.72, p. 197-230, 2000.

INEP. Censo da Educação Superior 2018. Brasília: ME/ INEP. 2019.

LAPLANE, Adriana Lia Friszman de. A inclusão escolar na Inglaterra. In: MENDES, Enicéia Gonçalves; ALMEIDA, Maria Amélia. (Org.). Das margens ao centro: perspectivas para as políticas e práticas educacionais no contexto da educação especial inclusiva. Araraquara: Junqueira-Marin, 2010. p. 19-30.

LÓPEZ-GAVIRA, Rosario; MORIÑA-DÍEZ, Anabel. Voces ocultas en la educación superior: políticas y prácticas inclusivas en las aulas de Ciencias Sociales y Derecho. International Journal of Inclusive Education, 19(4), p. 365-378, 2015. 
MORIÑA-DÍEZ, Anabel; CARBALLO, Rafael. Universidad y educación inclusiva: recomendaciones desde la voz de estudiantes españoles con discapacidad. Educ. Soc. [online]. 2020, vol.41, p. 1-16. May 11, 2020.

OMOTE, Sadao. Atitudes em Relação à Inclusão no Ensino Superior. Journal of Research in Special Educational Needs, v. 16, p. 211-215, 2016.

SEBASTIAN-HEREDERO, Eladio. A escola inclusiva e estratégias para fazer frente a ela: as adaptações curriculares. Acta Scientiarum Education. Maringá, v. 32, n. 2, p. 193208, 2010.

SILVA, Marcela Ribeiro Da; CAMARGO, Eder Pires De. O processo de inclusão no Ensino Superior: relatos e reflexões sobre a experiência de dois licenciados em Física com deficiência visual na universidade. In: MOL, Gerson de Souza; MELO, Douglas Christian Ferrari de. (Org.). Pessoas com deficiência no ensino superior: Desafios e possibilidades. Brasil: Multicultural, 2018. p. 108-121.

UNESCO. Conferencia Mundial sobre Educación Superior para el siglo XXI: Visión y acción. Paris: UNESCO, 1998.

UNESCO. Conferencia mundial sobre la educación superior - 2009: la nueva dinámica de la educación superior y la investigación para el cambio social y el desarrollo. Paris: UNESCO, 2009.

UNIVERSIA. IV Estudio sobre el grado de inclusión del sistema universitario español respecto de la realidad de la discapacidad. Madrid: Fundación Universia. 2018. Disponível em: https://www.fundacionuniversia.net/wpcontent/uploads/2019/05/IVEstudio_UniversidadyDiscapacidad_ACC.pdf Acesso em: 23 abr. 2020.

VELOSO, Teresa Cristina Mertens Aguiar; MACIEL, Carina Elizabeth. Acesso e permanência na educação superior - análise da legislação e indicadores educacionais. Revista Educação em Questão, Natal, v. 51, n. 37, p. 224-250, jan./abr. 2015.

\section{Notas}

${ }^{1}$ Utilizamos aquí el término personas con discapacidad por ser éste el utilizado por la comunidad científica en países de lengua española en base a las discusiones actuales y las políticas desarrolladas, aunque comprendemos que en Brasil el uso de personas con deficiencia es el utilizado en base a la Convención Universal de la Persona con Deficiencia.

\section{Correspondência}

Eladio Sebastián-Heredero - Universidade Federal de Mato Grosso do Sul. Programa de Pós-graduação em Educação. FAED. Avda UFMS s/n .79070-900. Campo Grande (MS) Brasil.

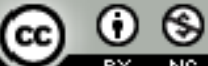

4.0 International (CC BY-NC 4.0) 